\title{
Investigation of harmonic components on voltage flicker measurement
}

\begin{abstract}
Harmonics and voltage flicker are increasing dramatically in power system due to wide use of non-linear loads. Specifically, voltage flicker is detected due to fluctuation of Root-MeansSquare (rms) value. Meanwhile, harmonics with certain components may lead to fluctuation of rms value too. Then, harmonic components may give significant impact on voltage flicker measurement. Therefore, this paper points out a worthwhile research gap regarding investigation of harmonic components on voltage flicker measurement. For further validation of the work, various harmonic orders and percentage injections generated from programmable AC source are analyzed by flickermeter (Fluke power analyzer 435 series II). From the findings, it is confirmed that second order harmonic component gives the most significant impact on voltage flicker measurement. Most importantly, a new linear expression is formed to clearly describe relationship between Short Term Perceptibility (Pst) and percentage of second harmonic component.
\end{abstract}

Keyword: Voltage flicker; Second harmonic; Power quality; Root-means-square (rms) 